\title{
Rheological Studies of Wheat Flour Dough
}

\section{Part I. Measurement of Dynamic Visco-elasticity}

\author{
By Toru Shimizu and Akiyoshi IchibA \\ Central Laboratory of Nisshin Flour Milling Co.
}

Received May 10, 1958

\begin{abstract}
Dynamic rigidity $G^{\prime}$, dynamic viscosity $\eta^{\prime}$ and the relaxation spectrum $L(t)$ of dough under periodic shear stress have been obtained. At first, $G^{\prime}$ and $\eta^{\prime}$ decreased rapidly with rest time, but maintained a constant value after 60 minutes. Values of both $G^{\prime}$ and $\eta^{\prime}$ decreased, and $L(t)$ became flat with the decrease in water absorption and with the temperature. $G^{\prime}$ increased slightly with the increase in salt content, but $\eta^{\prime}$ decreased in a low frequency range.
\end{abstract}

\section{INTRODUCTION}

The rheological behavior of wheat flour dough has been considered to be a more important field of research in cereal chemistry. Schofield and Scott Blair ${ }^{1}$ have adopted dough as a typical example of soft materials and have pointed out its intricate mechanical behaviors. And, many investigators have obtained numerous data using practical instruments, for example, such instruments as the Farinograph, Extensograph and Alveograph. Details of these have been reviewed by Bailey ${ }^{21}$ and more recently by Greup, Hintzer ${ }^{3}$ and Aitken ${ }^{4}$.

Recently, Cunningham, HIynka and Anderson $^{5,6,7)}$ have devised a relaxometer, and measured the stress relaxation of dough by application of this instrument. The measurement of visco-elasticity may be divided in static and dynamic methods. Stress relaxation is a

1) R. K. Schofield and G. W. Scott Blair, Proc. Roy. Soc. A. 138, 707 (1932);A. 139, 557 (1933); A. 141, 72 (1933); A.160, 87 (1937).

16, $242(1940)$

3) D. H. Greup and H. M. R. Hintzer, "Foodstuffs" ed. by Scott Blair, North Holland Pub. (1953).

4) T. R. Aitken, A. A.C.C. Trans, 12, 157 (1954).

5) J.R. Cunningham and I. Hlyoka, and J.A. Anderson, Can. 6) J. R. Cunningham and I. Hlynka, J. Appl. Pbys., 25,

7) I. Hlynka and J. A. Anderson, Agr. \& Food Chem., 5, 56 typical example of the static method. On the other hand, Slater ${ }^{83}$ has illustrated a method by which the dynamic property of concentrated flour-water suspension has been measured. In order to observe dynamic visco-elasticity of dough under periodic shear stress, the authors have modified the method described by Nakagawa and Seno $\widetilde{o}^{9)}$ This method is adapted to a low frequency range: for the purpose of obtaining an over-all relaxation spectrum, it is necessary to use it in combination with other methods. But when the bread making process is taken into consideration, rheological behavior in the low frequency range would be especially useful.

\section{EXPERIMENTAL}

1. Apparatus. A sketch of the apparatus is given in Figure 1. Dough (11) is set in a cylindrical cup (5) (radius $r_{1}=2.84 \mathrm{~cm}$ ) which is supported by a stand (7) and put in a thermoregulated water-bath (6). A cylindrical bob (4) (radius $r_{2}=2.00 \mathrm{~cm}$ ), whose end is cone-shaped, is inserted into the center of the dough. The bob is suspended by a piano wire (3) and a driving device (1) gives an oscillation $\Theta=\Theta_{0} \sin \omega t$ to the top of the wire (2). The bob, which is inserted in the visco-elastic body, shows a different oscillation $\theta=A \sin (\omega t-\phi)$. To measure the amplitude ratio $p=$

8) L. E. Slater, Food Eng., 26, May 74 (1954).

9) T. Nakagawa \& M. Senô, Bull. Chem. Soc. Japan, 29, 
$A / \Theta_{0}$ and the phase difference $\phi$, the optical Lissajous' method was adopted.

Small galvanometer mirrors $\mathbf{M}_{1}$ and $\mathbf{M}_{2}$ are fixed to the top of the piano wire, and to the bob. A convex lens $L$ is placed in front of $M_{1}$ and $M_{2}$. Light emitted from the source at (8) is made parallel by $L$, is reflected by $M_{1}$ and $M_{2}$, and is then converged by $L$. A strip mirror $\mathrm{M}_{3}$ is placed at an angle of $45^{\circ}$ to the beam of light in the camera (9). $\mathbf{M}_{3}$ is connected to the oscillation mechanism and a photographic paper (10) is placed at the focus of L. Two Lissajous' figures, the one a linear line (D) and the other an elliptic curve (E) from two oscillations whose directions are at right angles to each other, are obtained. A typical Lissajous' figure is shown in Figure 2.

2. Calculations. Equation of motion of the bob is expressed as follows:

$$
\ddot{I} \theta=-k_{0} \theta-R \dot{\theta}-K \theta
$$

where, I Moment of inertia of the bob. $k_{0}$ Torison constant of the piano wire

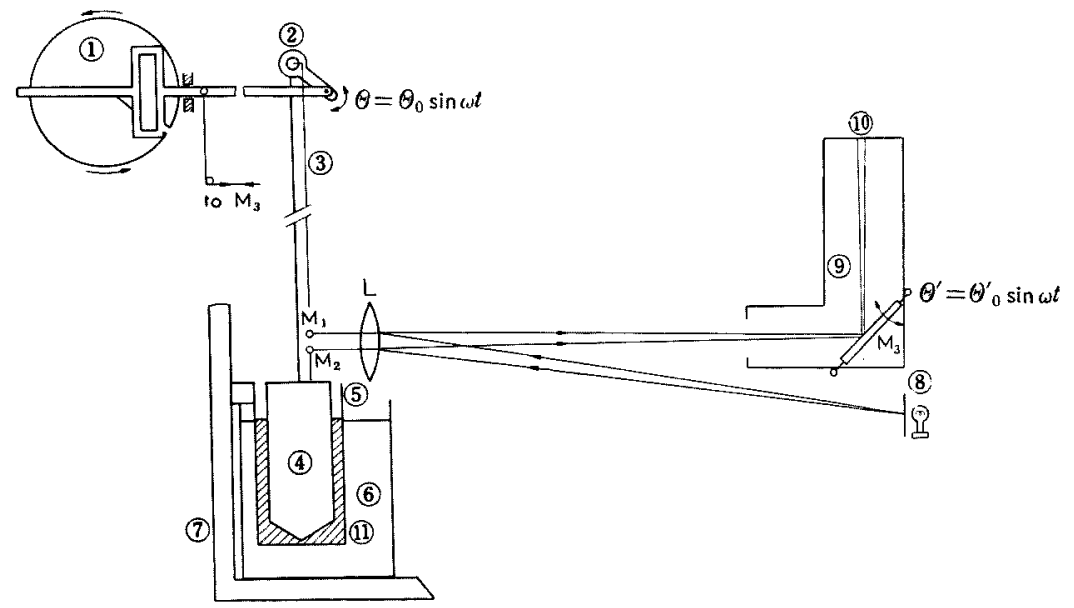

FIG. 1. Schematic diagram of the instrument.

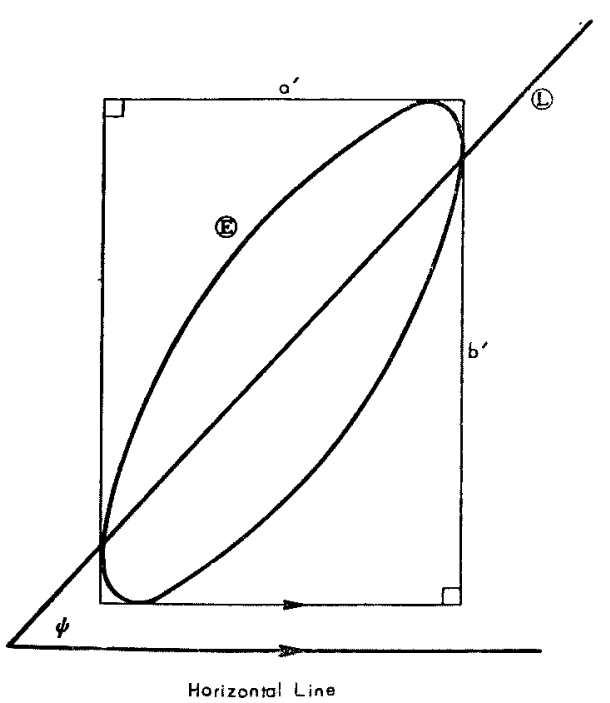

FIG. 2. Typical Lissajous' figures.
$R$ Resistance of dough.

$K$ Elastance of dough.

Nakagawa and Senô ${ }^{9)}$ have indicated a relationship between the data obtained from the Lissajous' figure and the solution of Equation (1), which is as follows:

$$
\left.\begin{array}{rl}
\sin \phi & =\begin{array}{l}
4(S) \\
\pi[S] \\
p
\end{array}=\frac{b^{\prime}}{a^{\prime}} \tan \phi \\
K & =k_{0}\left(\frac{\cos \phi}{p}-1\right)+\frac{4 \pi^{2} I}{T_{f^{2}}} \\
R & =\frac{k_{0} \cdot T_{F} \cdot \sin \phi}{2 \pi p}
\end{array}\right\} \quad(3)
$$




$$
\begin{aligned}
& \text { parallel with the horizontal line and each side } \\
& \text { is tangent to the curve (See Fig. 2). } \\
& \text { Angle between } L \text { and the horizontal line. } \\
& \text { Depth of dough. }
\end{aligned}
$$

The real and imaginary part $G^{\prime}, G^{\prime \prime}$ of complex dynamic modulus $G^{*}$ can be expressed by the relaxation spectrum $L(t)$ as follows:

$$
\left.\begin{array}{rl}
G^{\prime} & =\int_{-\infty}^{+\infty} \frac{t_{1}^{2} \tau^{2}}{1+\omega^{2} \tau^{2}} L(t) d \log \tau \\
\eta^{\prime} & =\int_{-\infty}^{+\infty} \frac{\omega \tau}{1+r \nu^{2} \tau^{2}} L(t) d \log \tau
\end{array}\right\}
$$

Schwarz ${ }^{10)}$ has indicated a method by which $L(t)$ is obtained approximately as follows :

$$
\left.\begin{array}{rl}
L(t) & =\frac{2}{\pi}\left[G^{\prime \prime}(\omega)\right]_{\omega=\tau^{-1}} \\
G^{\prime \prime}(\omega) & =\omega \eta^{\prime}(\omega)
\end{array}\right\}
$$

The following data were obtained with the aid of of Equations (2), (3), (4), (6).

3. Material and Experimental Procedure. A long patent flour obtained from Canadian Manitoba Northern \#3 wheat was used in this study. The flour contained $0.41 \%$ ash and $11.3 \%$ crude protein on a $14 \%$ moisture basis. Dough was prepared by mixing the flour in Kenwood mixer for $1.5 \mathrm{~min}$.. A hundred grams of dough was rounded by a Brabender Extensograph rounder 20 times and set in the cup. After a constant rest period, a forced oscillation (Amplitude, 0.062 radian) was given to the top of the piano wire. The diameter of the wire was $2.4 \mathrm{~mm}$ and the torsion constant was $3.65 \times 10^{6}$ dyne.cm.

\section{RESULTS AND DISCUSSION}

1. Changes of $\boldsymbol{G}^{\prime}, \eta^{\prime}$ with Rest Time. Results are shown in Figure 3. The experimental conditions were;

Temperature $28^{\circ} \mathrm{C}$, Water absorption $63 \%$, $66 \%$ Period 2.7 sec.

At first, both $G^{\prime}$ and $\eta^{\prime}$ of dough decreased rapidly, but maintained a constant value after about 60 minutes. It is thought that these results are related to the structural relaxation of dough which has been reviewed by Hlynka and Anderson ${ }^{7}$. From these results, a rest time of 60 minutes was selected for the following experiments.

2. Changes of Rheological Properties at different Water Absorptions. $G^{\prime}, \eta^{\prime}$ and $L(t)$ of $63 \%$ and $66 \%$ water absorption are shown in Figure 4. Data represent the function of period. The 10) F. Schwarzl, Inter. Cong. Rheology, 1953, 197.

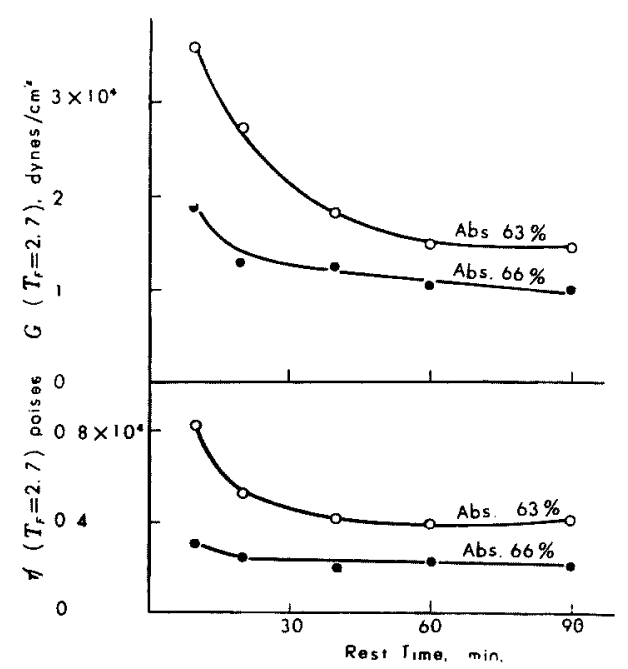

FIG. 3. Changes of $G^{\prime}$ and $\eta^{\prime}$ with rest time.

experimental conditions were;

Temperature $28^{\circ} \mathrm{C}$, Rest time $60 \mathrm{~min}$., Period 1.7, 2.7, 10.1, 23.5 sec. .

$G^{\prime}$ and $\eta^{\prime}$ decreased and $L(t)$ became flat with the increase in water absorption. These results correspond to those of Halton and Scott

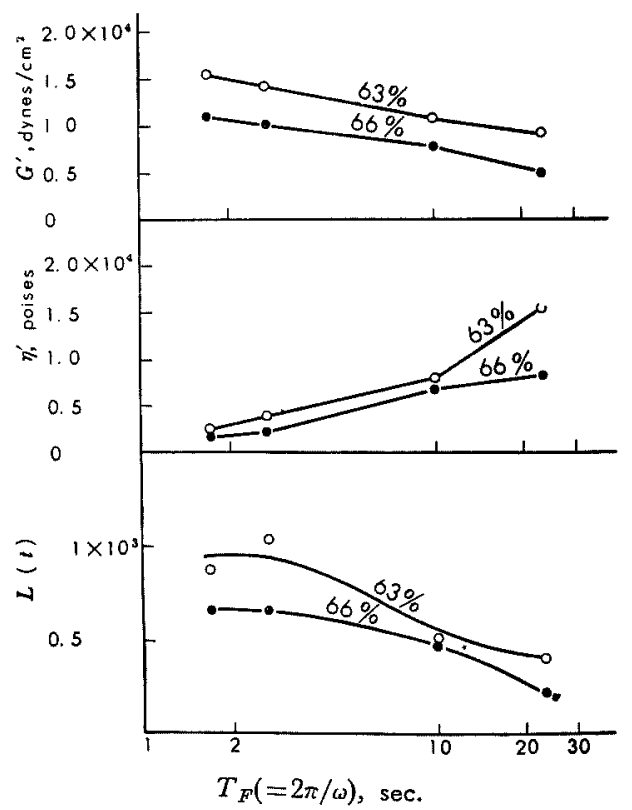

FIG. 4. Changes of $G^{\prime}, \eta^{\prime}$ and $L(t)$ at different water absorptions. 


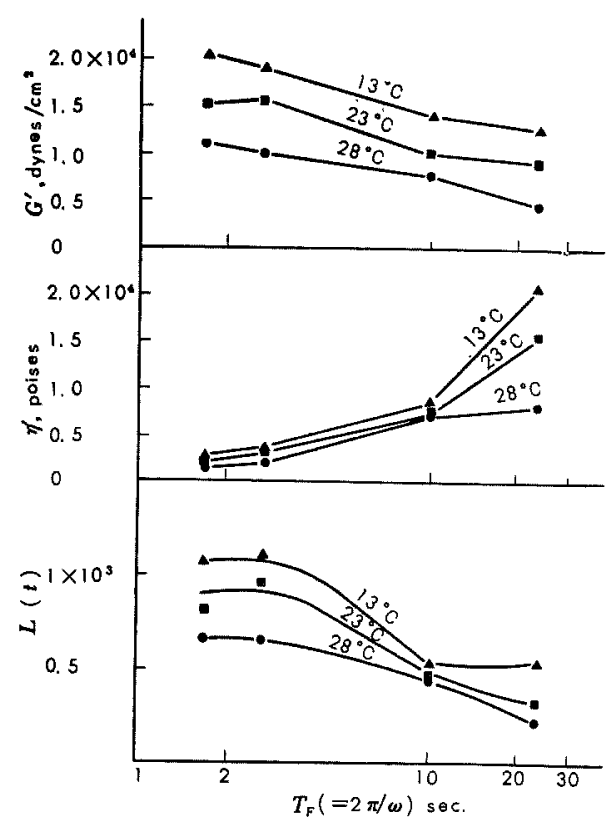

Fig. 5. Changes of $G^{\prime}, \eta^{\prime}$ and $L(t)$ at different temperatures.

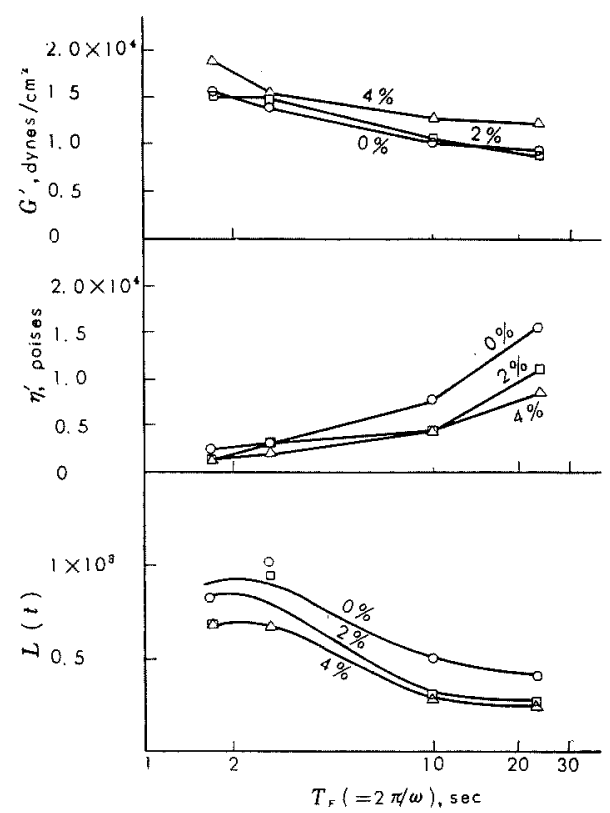

FIG. 7. Changes of $G^{\prime}, \eta^{\prime}$ and $L(t)$ at different salt contents.

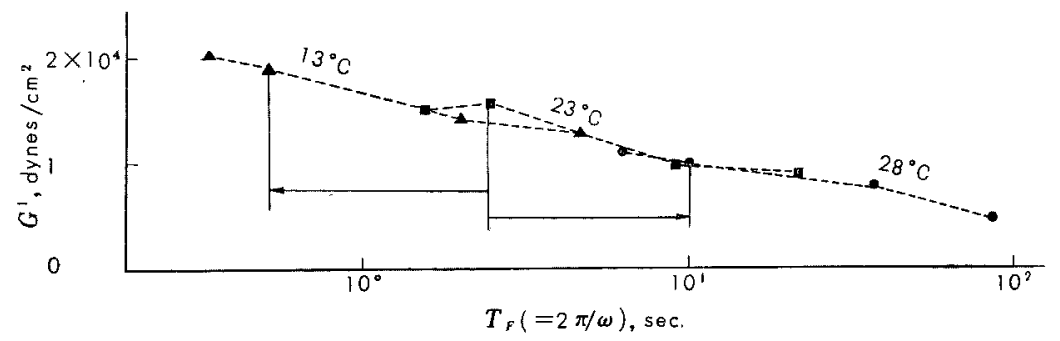

Fig. 6. Shift of Figure 5.

Blair $^{11)}$ and Cunningham and Hlynka ${ }^{6)}$ obtained in static experiments.

3. Changes of Rheological Properties at Different Temperatures. $G^{\prime}, \eta^{\prime}$ and $L(t)$ at $13^{\circ} \mathrm{C}$, $23^{\circ} \mathrm{C}$ and $28^{\circ} \mathrm{C}$ are shown in Figure 5 . Water absorption was $66 \%$ and other experimental conditions were the same as in the former experiment.

$G^{\prime}$ and $\eta^{\prime}$ decreased and $L(t)$ became flat with the increase in temperature. When the $G^{\prime}-\log . T_{F}$ relationship at $13^{\circ} \mathrm{C}$ was shifted

11) P. Halton and G.W. Scott Blait, Cereal Chem., 14, 201 (1937). to the left along the time axis and the relationship at $28^{\circ} \mathrm{C}$ to the right, they formed part of a master curve as that in Figure 6. From this fact, it is suggested that flour dough is approximately thermorheologically simple as many high polymers and therefore the data in the low temperature range can be used instead of that in the high frequency range.

4. Changes of Rheological Properties at Different Salt Content. $G^{\prime}, \eta^{\prime}$ and $L(t)$ at zero, 2 and $4 \%$ salt content to Hlour are shown in Figure 7. Water absorption was $63 \%$ and other experimental conditions were the same as these 
in the former experiment.

$G^{\prime}$ increased slightly with the increase in salt content, but $\eta^{\prime}$ decreased in the low frequency range. As it is anticipated that inorganic ions have a stroug influence on the rheological properties of dough, this problem will be the subject of future investigation.

These results lead to the following comments. 1) If a four parameter mechanical model can be assumed for dough, the relation between static and dynamic rigidity may readily be expressed as :

$$
G^{\prime}=G_{1}+G_{2} \frac{\omega^{2} \tau_{2}^{2}}{1+\omega^{2} \tau_{2}^{2}} \quad\left(\tau_{2}=\eta_{2} / G_{2}\right)
$$

As elasticity, which has been obtained by Halton and Scott Blair ${ }^{10)}$, is a mixture of instantaneous rigidity $G_{1}$ and retarded rigidity $G_{2}$, the order of magnitude $G^{\prime}$ of the present experiments is the same as that of the former data.
2) Accuracy of the approximation by which relaxation spectrum is obtained, is in the same order magnitude as that of Cunningham and Hlynka $^{6)}$ Moreover, this approximation method is very simple.

3) In contrast to data obtained from dough testing machines, the authors can obtain the data in c.g.s. units by this method. It is probable that these data are comparable with those of other materials other than wheat flour dough.

Acknowledgement. The authors wish to express their sincere thanks to Dr. T. Nakagawa of Tokyo University for his constant guidance, and to Dr. I. Hlynka of the Grain Research Laboratory, Winnipeg, Canada, for his kind advice. They also wish to thank Mr. H. Shoda, president of Nisshin Flour Milling Co., and Mr. T. Yasui, director in charge of this laboratory, for his encouragement in this work. 